\title{
GAUSSIAN HARMONIC FORMS AND TWO-DIMENSIONAL SELF-SHRINKING SURFACES
}

\author{
MATTHEW MCGONAGLE
}

(Communicated by Michael Wolf)

\begin{abstract}
We consider two-dimensional self-shrinkers $\Sigma^{2}$ for the Mean Curvature Flow of polynomial volume growth immersed in $\mathbb{R}^{n}$. We look at closed one forms $\omega$ satisfying the Euler-Lagrange equation associated with minimizing the norm $\int_{\Sigma} d V e^{-|x|^{2} / 4}|\omega|^{2}$ in their cohomology class. We call these forms Gaussian Harmonic one Forms (GHF).

Our main application of GHF's is to show that if $\Sigma$ has genus $\geq 1$, then we have a lower bound on the supremum norm of $|A|^{2}$. We also may give applications to the index of $L$ acting on scalar functions of $\Sigma$ and to estimates of the lowest eigenvalue $\eta_{0}$ of $L$ if $\Sigma$ satisfies certain curvature conditions.
\end{abstract}

\section{INTRODUCTION}

A two-dimensional submanifold $\Sigma^{2}$ immersed in $\mathbb{R}^{n}$ is considered a self-shrinker for the Mean Curvature Flow if it satisfies

$$
H=\frac{x^{N}}{2}
$$

(note our choice of normalization) where $x$ is the position vector in $\mathbb{R}^{n}$. Selfshrinkers are related to the singularities of the Mean Curvature Flow [2]. For this paper, we will only consider $\Sigma$ to be a complete two-dimensional self-shrinker of polynomial volume growth.

For a properly immersed self-shrinker $\Sigma$, we expect bounds on the curvature to affect the topology of $\Sigma$. Let $A$ denote the second fundamental form of $\Sigma$. An argument, pointed out to the author by Professor Minicozzi, shows that if $|A|^{2} \leq C|x|^{2}$ with $C<1 / 8$ for $|x|$ large, then $\Sigma$ has finite topology. For any twodimensional $\Sigma$ we have $|H|^{2} \leq 2|A|^{2}$. Since we are on a self-shrinker, we then have that $\left|x^{N}\right|^{2} \leq 8 C|x|^{2}$. We then have that $\left|x^{T}\right|^{2} \geq(1-8 C)|x|^{2}>0$ for $|x|$ sufficiently large. Therefore, we may apply Morse Theory to find that the topology is constant for large enough $|x|$.

In Ros [6] and Urbano [7] harmonic one forms are used to study the index of a one-sided minimal surface of genus $g$. The harmonic forms are constructed by minimizing the $L^{2}$ norm $\int_{\Sigma} d V|\omega|^{2}$ in a cohomology class. From classical Riemann surface theory [3, p. 42], we can associate $2 g$ linearly independent $L^{2}$ harmonic one forms to a surface of genus $g$. Ros [6] and Urbano [7] consider the duals to these

Received by the editors January 2, 2013.

2010 Mathematics Subject Classification. Primary 53A10, 53C42; Secondary 53C44.

Key words and phrases. Mean Curvature Flow, self-shrinkers, harmonic one forms, genus, Gaussian harmonic. 
harmonic one forms as vectors in Euclidean space. They study the Jacobi operator $\triangle_{\Sigma}+|A|^{2}$ acting on the coordinate functions of these vectors.

We consider a parallel situation for a self-shrinker $\Sigma$. We examine closed one forms $\omega$ minimizing $\int_{\Sigma} d V e^{-|x|^{2} / 4}|\omega|^{2}$ in their cohomology class. Any closed one form satsifying the Euler-Lagrange Equation for this minimization will be called a Gaussian Harmonic one Form (GHF). Note that this norm does not come from a conformal change of $\Sigma$. The measure $e^{-|x|^{2} / 4} d V$ is related to the variational characterization of self-shrinkers in Colding-Minicozzi [2]. A difference between this and the harmonic case is that we only get $g$ linearly independent GHF, because the Euler-Lagrange condition for GHF is not preserved by a rotation. For simplicity in notation, we will define $d \mu \equiv e^{-|x|^{2} / 4} d V$. We will also denote $L_{\mu}^{2}(\Sigma)$ to be the $L^{2}$ space associated with the measure $d \mu$.

For a normal vector field $N$ and tangent vector fields $X, Y$ we define the Second Fundamental Form by $A^{N}(X, Y)=\left\langle\nabla_{X}^{E} N, Y\right\rangle$. We also define $B(X, Y)=-\nabla_{X}^{N} Y$ and $H=B(i, i)$. Our sign convention is chosen to be consistent with that of Colding-Minicozzi 2].

We will use $K$ to refer to the curvature of $\Sigma$. For an orthonormal frame $N_{\alpha}$ in the normal bundle $N \Sigma$, Gauss' Equation gives us that $K=A_{11}^{N_{\alpha}} A_{22}^{N_{\alpha}}-A_{12}^{N_{\alpha}} A_{12}^{N_{\alpha}}$.

For each $N_{\alpha}$, we may diaganolize $A^{N_{\alpha}}$ with eigenvalues $\kappa_{\alpha i}$ for a frame $\left\{e_{\alpha i}\right\}$ in $T \Sigma$. Note, that from Gauss' Equation we have that $K=\frac{1}{2}\left(A_{i i}^{N_{\alpha}} A_{j j}^{N_{\alpha}}-A_{i j}^{N_{\alpha}} A_{i j}^{N_{\alpha}}\right)$, and so $K=\sum_{\alpha} \kappa_{\alpha 1} \kappa_{\alpha 2}$. We denote the components of a tangential vector field $X$ with respect to $e_{\alpha i}$ as $X_{\alpha i}$.

We will have need to work with vectors in $T \Sigma, N \Sigma$, and in $\mathbb{R}^{n}$. We will use the indices $\{i, j, \ldots\}$ for vectors or forms considered intrinsically part of $\Sigma,\{a, b, \ldots\}$ when considering vectors in $\mathbb{R}^{n}$, and $\{\alpha, \beta, \ldots\}$ when considering vectors in $N \Sigma$.

The vectors $\partial_{a}$ will denote a standard basis of orthonormal vectors for $\mathbb{R}^{n}$. We will make use of the exterior derivative of the coordinate function $x^{a}$ along $\Sigma$ and will denote this by $d x^{a}$. That is, $d x^{a}$ is not the exterior derivative in $\mathbb{R}^{n}$. Also, for any vector $V \in \mathbb{R}^{n}$ let $V^{T}$ be the projection onto the tangent space of $\Sigma$. For any one form $\omega$ on $\Sigma$ we shall denote the vector dual to $\omega$ considered to be sitting in $\mathbb{R}^{n}$ as $W$.

As in Colding-Minicozzi [2], we define the operators $\mathcal{L}$ and $L$ on scalar functions of $\Sigma$ as

$$
\begin{aligned}
& \mathcal{L} u \equiv \Delta u-\frac{1}{2} \nabla_{x^{T}} u \\
& L u \equiv \mathcal{L} u+\frac{1}{2} u+|A|^{2} u .
\end{aligned}
$$

Note that $\mathcal{L}=-\nabla^{*} \nabla$ for the measure $d \mu$. That is, $\int_{\Sigma} d \mu f \mathcal{L} g=-\int_{\Sigma} d \mu\langle\nabla f, \nabla g\rangle$ for any $f, g \in C_{0}^{\infty}(\Sigma)$. In particular, $\mathcal{L}$ is a symmetric operator. The operator $L$ is associated with the second variation related to the variational characterization of self-shrinkers in Colding-Minicozzi [2].

Since we will be working with vectors in $T \Sigma, N \Sigma$, and $\mathbb{R}^{n}$ we will use $\nabla^{E}$ to denote the Euclidean connection, $\nabla^{\Sigma}=\left(\nabla^{E}\right)^{T}$ for the connection on $\Sigma$, and $\nabla^{N}=\left(\nabla^{E}\right)^{N}$ for the normal connection. 
We also define the operators $\mathcal{L}^{E}$ and $L^{E}$ on $\mathbb{R}^{n}$ valued vector fields $V$ along $\Sigma$ by

$$
\begin{aligned}
\mathcal{L}^{E} V & \equiv \operatorname{Tr}_{\Sigma} \nabla^{2, E} V-\frac{1}{2} \nabla_{x^{T}}^{E} V \\
& =\nabla_{i, i}^{2, E} V-\frac{1}{2} \nabla_{x^{T}}^{E} V \\
L^{E} V & \equiv \mathcal{L}^{E} V+\frac{1}{2} V+|A|^{2} V .
\end{aligned}
$$

Note that for $V=V^{a} \partial_{a}$ we have $\mathcal{L}^{E} V=\left(\mathcal{L} V^{a}\right) \partial_{a}$ and $L^{E} V=\left(L V^{a}\right) \partial_{a}$.

We will likewise use the $\nabla^{\Sigma}$ connection to define $\mathcal{L}^{\Sigma}$ and $L^{\Sigma}$ on tensors intrinsic to $\Sigma$.

In the first section of the paper, we establish some computations for GHF's on $\Sigma$. The main results of our computations are (1.8) and (1.9).

For the second section, we apply our computations for GHF's to prove two results in the general co-dimension case. The first is a type of "gap theorem" for the genus of a self-shrinker.

Theorem 2.1. If $\Sigma$ is a two-dimensional orientable self-shrinker of polynomial volume growth immersed in $\mathbb{R}^{n}$ with genus $\geq 1$, then

$$
\sup _{x \in \Sigma,|v|=1} A^{N_{\beta}}(v, i) A^{N_{\beta}}(i, v) \geq 1 / 2 .
$$

Remark. In the case of $\Sigma \rightarrow \mathbb{R}^{3}$ we have that $\sup _{x \in \Sigma,|v|=1} A^{N_{\beta}}(v, i) A^{N_{\beta}}(i, v)=\sup _{x \in \Sigma, i} \kappa_{i}^{2}$ where the $\kappa_{i}$ are the principal curvatures of $\Sigma$. Theorem 3.1 should also be compared with a result of Cao-Li [1], where they prove a gap theorem for self-shrinkers $\Sigma^{k} \subset$ $\mathbb{R}^{n}$ of any co-dimension. They show that if the square norm $|A|^{2} \leq 1 / 2$ (here we have renormalized their result for our definition of self-shrinker), then $\Sigma^{k}$ is a round sphere, a cylinder, or a hyperplane.

Next, we show a lower bound on the index of $L$ based on the genus of $\Sigma$ if $\Sigma$ satisfies an appropriate curvature condition.

Theorem 2.2. Let $\Sigma$ be a two-dimensional orientable self-shrinker of polynomial volume growth immersed in $\mathbb{R}^{n}$ with genus $g$ and principal curvatures $\kappa_{\alpha i}$ for a normal frame $\left\{N_{\alpha}\right\}$. If $\sup _{p \in \Sigma} \inf _{\left\{N_{\alpha}(p)\right\}} \sum_{\alpha}\left|\kappa_{\alpha 1}^{2}-\kappa_{\alpha 2}^{2}\right| \leq \delta<1$, then the index of $L$ acting on scalar functions of $\Sigma$ has a lower bound given by

$$
\operatorname{Index}_{\Sigma}(L) \geq \frac{g}{n}
$$

Remark. Note, that in the case of $\Sigma \subset \mathbb{R}^{3}$, we get that $\sup _{p \in \Sigma} \inf _{\left\{N_{\alpha}(p)\right\}} \sum_{\alpha}\left|\kappa_{\alpha 1}^{2}-\kappa_{\alpha 2}^{2}\right|=$ $\sup \left|\kappa_{1}^{2}-\kappa_{2}^{2}\right|$.

In the third section, we make applications to the lowest eigenvalue of $L$ acting on scalar functions of $\Sigma$. Our first result is for the case where $\Sigma$ isn't necessarily compact. 
Theorem 3.1. Let $\Sigma$ be a two-dimensional orientable self-shrinker of polynomial volume growth immersed in $\mathbb{R}^{n}$ with genus $\geq 1$. The lowest eigenvalue of $L$ acting on scalar functions on $\Sigma$ has upper bounds given by

$$
\begin{aligned}
& \eta_{0} \leq-1+\sup _{x \in \Sigma,|v|=1} A^{N_{\beta}}(v, i) A^{N_{\beta}}(i, v) \\
& \eta_{0} \leq-1+\sup _{p \in \Sigma} \inf _{\left\{N_{\alpha}(p)\right\}} \sum_{\alpha}\left|\kappa_{\alpha 1}^{2}-\kappa_{\alpha 2}^{2}\right| .
\end{aligned}
$$

Remark. From Theorem 3.1 we see that the best upper bound (0.9) can give us is $\eta_{0} \leq-1 / 2$. In the co-dimension one case $\Sigma \rightarrow \mathbb{R}^{3}$, Colding-Minicozzi [2] were able to show that $\eta_{0} \leq-1$ by showing that $L H=H$ and that $H$ is in the appropriate weighted space. So, we see that the estimate of Theorem 3.2 isn't optimal for the codimension one case. For the cases of higher co-dimension, one still has $L^{N} H=H$, but this doesn't say anything about the eigenvalues of $L$ on scalar functions unless there exists a global parallel normal.

Then, we show an estimate for the lowest eigenvalue of $L$ for compact $\Sigma$. We get a better bound since the compactness of $\Sigma$ gives us that $\eta_{0}$ is realized by an eigenfunction. This allows us to use $\mathcal{L}^{\Sigma}$ instead of $\mathcal{L}^{E}$ to give a better estimate.

Theorem 3.2. Let $\Sigma$ be a two-dimensional orientable compact self-shrinker immersed in $\mathbb{R}^{n}$ with genus $g \geq 1$ and principal curvatures $\kappa_{\alpha i}$ in a normal frame $\left\{N_{\alpha}\right\}$. Let $\eta_{0}$ be the lowest eigenvalue of $L$ acting on scalar functions on $\Sigma$. We have that

$$
\eta_{0} \leq-3 / 2+\sup _{p \in \Sigma} \inf _{\left\{N_{\alpha}(p)\right\}} \sum_{\alpha}\left|\kappa_{\alpha 1}^{2}-\kappa_{\alpha 2}^{2}\right|
$$

Remark. As before, we note that Colding-Minicozzi 2 have shown that in the codimension one case that $\eta_{0} \leq-1$. Therefore, we see that (0.11) is only optimal for $\Sigma^{2} \subset \mathbb{R}^{3}$ if $\left|\kappa_{1}^{2}-\kappa_{2}^{2}\right|<1 / 2$.

\section{Gaussian Harmonic one Forms}

Let $\omega_{0}$ be a closed $C_{0}^{\infty}$ one form on $\Sigma$, i.e., $d \omega_{0}=0$. The classical harmonic form cohomologous to $\omega_{0}$ is constructed by minimizing the $L^{2}(\Sigma)$ norm $\int_{\Sigma}|\omega|^{2}$ in the cohomology class of $\omega_{0}$ [5]. A form $\omega$ is defined to be harmonic if and only if it is closed $\left(\nabla^{\Sigma} \omega\right.$ is symmetric) and co-closed $\left(\nabla^{\Sigma} \omega\right.$ is traceless) 6.

Instead of using harmonic one forms to represent the cohomology class of $\omega_{0}$ as in Ros [6] and Urbano [7, we will use the form minimizing the $L_{\mu}^{2}(\Sigma)$ norm $\int_{\Sigma} d \mu|\omega|^{2}$ where $\omega=\omega_{0}+d f$ and $d \mu=e^{-|x|^{2} / 4} d V$. To find the Euler-Lagrange Equation, assume the minimum is achieved by a closed form $\omega$. Also, let $\delta$ be the dual to $d$ with respect to the regular Euclidean surface measure $d V$. As in Jost [5], the minimization gives us that $0=\delta\left(e^{-|x|^{2} / 4} \omega\right)=-\operatorname{div}\left(e^{-|x|^{2} / 4} \omega\right)$. Using this, we obtain the Euler-Lagrange Equation:

$$
\operatorname{Tr}_{\Sigma} \nabla^{\Sigma} \omega=\frac{1}{2} \omega\left(x^{T}\right)
$$

We make a definition:

Definition 1. A form $\omega$ will be called a Gaussian Harmonic Form (GHF) if and only if $\omega$ is closed ( $\nabla^{\Sigma} \omega$ is symmetric) and $\omega$ is Gaussian co-closed (satisfies 1.1). 
We have the following result:

Lemma 1.1. Let $\omega$ be a GHF on a self-shrinker $\Sigma^{2}$ with dual vector $W \in T \Sigma$. We have that

$$
\left(\mathcal{L}^{\Sigma} \omega\right)(v)=\frac{1}{2} \omega(v)-A^{N_{\alpha}}\left(W, e_{i}\right) A^{N_{\alpha}}\left(e_{i}, v\right) .
$$

Proof. We have a Weitzenbock formula $\triangle^{\Sigma} \omega=-(\delta d+d \delta) \omega+K \omega$ where $\delta d+d \delta$ is the Hodge Laplacian [5]. Now $\delta \omega=-\operatorname{div} \omega=-\frac{1}{2} \omega\left(x^{T}\right)$ and $d \omega=0$. Therefore $-(\delta d+d \delta) \omega=\frac{1}{2} d\left(\omega\left(x^{T}\right)\right)=\frac{1}{2} \nabla^{\Sigma}\left(\omega\left(x^{T}\right)\right)$. Then, using a Leibniz rule, we get

$$
\left(\triangle^{\Sigma} \omega\right)(v)=K \omega+\frac{1}{2} \omega\left(\nabla_{v}^{\Sigma} x^{T}\right)+\frac{1}{2} \nabla^{\Sigma} \omega\left(x^{T}, v\right) .
$$

Now, we use that for a self-shrinker, $\nabla_{v}^{\Sigma} x^{T}=v-2 A^{H}\left(v, e_{i}\right) e_{i}$ and that $\mathcal{L}^{\Sigma}=$ $\triangle^{\Sigma}-\frac{1}{2} \nabla_{x^{T}}^{\Sigma}$ to get

$$
\left(\mathcal{L}^{\Sigma} \omega\right)(v)=K \omega(v)+\frac{1}{2} \omega(v)-A^{H}(v, W) .
$$

Decomposing in a normal basis $\left\{N_{\alpha}\right\}$, we have that

$$
\begin{aligned}
K W-\sum_{i} A^{H}\left(W, e_{i}\right) e_{i} & =\sum_{\alpha} \sum_{i}\left(\kappa_{\alpha 1} \kappa_{\alpha 2} W_{\alpha i} e_{\alpha i}-\left(\kappa_{\alpha 1}+\kappa_{\alpha 2}\right) \kappa_{\alpha i} W_{\alpha i} e_{\alpha i}\right) \\
& =\sum_{\alpha} \sum_{i}-\kappa_{\alpha i}^{2} W_{\alpha i} e_{\alpha i} \\
& =-A^{N_{\alpha}}\left(W, e_{i}\right) A^{N_{\alpha}}\left(e_{i}, e_{j}\right) e_{j} .
\end{aligned}
$$

Note that the dual form of equation (1.2) is

$$
\mathcal{L}^{\Sigma} W=\left\langle\mathcal{L}^{\Sigma} \omega, d x^{a}\right\rangle \partial_{a}=\frac{1}{2} W-A^{N_{\beta}}\left(W, e_{j}\right) A^{N_{\beta}}\left(e_{j}, e_{k}\right) e_{k} .
$$

Lemma 1.2. Let $\omega$ be a GHF on a self-shrinker $\Sigma^{2}$. Let $W$ be the vector field dual to $\omega$. We have

$$
\mathcal{L}^{E} W=-2\left\langle\nabla^{\Sigma} \omega, A^{N_{\beta}}\right\rangle N_{\beta}+\frac{1}{2} W-2 A^{N_{\beta}}\left(W, e_{j}\right) A^{N_{\beta}}\left(e_{j}, e_{k}\right) e_{k} .
$$

Proof. First, we compute $\left\langle\nabla^{\Sigma} \omega, \nabla^{\Sigma} d x^{a}\right\rangle \partial_{a}$. Note that $\nabla^{\Sigma} \partial_{a}^{T}=-\left\langle\partial_{a}, N_{\beta}\right\rangle \nabla^{\Sigma} N_{\beta}$. Hence $\nabla^{\Sigma} d x^{a}=-N_{\beta}{ }^{a} A^{N_{\beta}}$, and so we get

$$
\left\langle\nabla^{\Sigma} \omega, \nabla^{\Sigma} d x^{a}\right\rangle \partial_{a}=-\left\langle\nabla^{\Sigma} \omega, A^{N_{\beta}}\right\rangle N_{\beta} .
$$

Next, we compute $\left\langle\omega, \mathcal{L}^{\Sigma} d x^{a}\right\rangle \partial_{a}$. Fix a point $p \in \Sigma$. Use a tangential frame $\left\{e_{j}\right\}$ and a normal frame $\left\{N_{\beta}\right\}$ such that $\nabla^{\Sigma} e_{j}(p)=0$ and $\nabla^{N} N_{\beta}(p)=0$. Using the Codazzi Equation, we get

$$
\begin{aligned}
\left(\triangle^{\Sigma} d x^{a}\right)\left(e_{j}\right) & =-A^{N_{\beta}}\left(\partial_{a}^{T}, e_{k}\right) A^{N_{\beta}}\left(e_{k}, e_{j}\right)-\left(\nabla_{e_{j}}^{N} H\right)^{a} \\
& =-A^{N_{\beta}}\left(\partial_{a}^{T}, e_{k}\right) A^{N_{\beta}}\left(e_{k}, e_{j}\right)-\frac{N_{\beta}{ }^{a}}{2} A^{N_{\beta}}\left(x^{T}, W\right) .
\end{aligned}
$$

Therefore,

$$
\left\langle\omega, \mathcal{L}^{\Sigma} d x^{a}\right\rangle \partial_{a}=-A^{N_{\beta}}\left(W, e_{j}\right) A^{N_{\beta}}\left(e_{j}, e_{k}\right) e_{k} .
$$


Now, for $W=\left\langle\omega, d x^{a}\right\rangle \partial_{a}$, we have

$$
\begin{aligned}
\mathcal{L}^{E} W & =\left(\mathcal{L}\left\langle\omega, d x^{a}\right\rangle\right) \partial_{a} \\
& =\left\langle\mathcal{L}^{\Sigma} \omega, d x^{a}\right\rangle \partial_{a}+2\left\langle\nabla^{\Sigma} \omega, \nabla^{\Sigma} d x^{a}\right\rangle \partial_{a}+\left\langle\omega, \mathcal{L}^{\Sigma} d x^{a}\right\rangle \partial_{a} .
\end{aligned}
$$

Using equations (1.2), (1.10), and (1.13), we get (1.9).

\section{Applications}

Theorem 2.1. If $\Sigma$ is a two-dimensional orientable self-shrinker of polynomial volume growth immersed in $\mathbb{R}^{n}$ with genus $\geq 1$, then

$$
\sup _{x \in \Sigma,|v|=1} A^{N_{\beta}}(v, i) A^{N_{\beta}}(i, v) \geq 1 / 2 .
$$

Proof. Assume $\Sigma$ has genus $g \geq 1$. Parallel to the result for classical Riemann surfaces [3], we then have $g$ linearly independent GHF in $L_{\mu}^{2}(\Sigma)$. Let $\omega$ be one of these GHF and $W$ be the dual to $\omega$.

Consider any $\phi \in C_{0}^{\infty}(\Sigma)$. We have

$$
\begin{aligned}
0 \leq \int_{\Sigma} d \mu\left|\nabla^{\Sigma}(\phi W)\right|^{2}= & -\int_{\Sigma} d \mu\left\langle\phi W, \mathcal{L}^{\Sigma}(\phi W)\right\rangle \\
= & -\int_{\Sigma} d \mu|W|^{2} \phi \mathcal{L} \phi-\frac{1}{2} \int_{\Sigma} d \mu\left\langle\nabla^{\Sigma} \phi^{2}, \nabla^{\Sigma}|W|^{2}\right\rangle \\
& -\int_{\Sigma} d \mu \phi^{2}\left\langle W, \mathcal{L}^{\Sigma} W\right\rangle .
\end{aligned}
$$

Now, using integration by parts, we have that

$$
-\frac{1}{2} \int_{\Sigma} d \mu\left\langle\nabla^{\Sigma} \phi^{2}, \nabla^{\Sigma}|W|^{2}\right\rangle=\int_{\Sigma} d \mu|W|^{2} \phi \mathcal{L} \phi+\int_{\Sigma} d \mu|W|^{2}\left|\nabla^{\Sigma} \phi\right|^{2} .
$$

Putting this into (2.3), we get that

$$
0 \leq \int_{\Sigma} d \mu|W|^{2}|\nabla \phi|^{2}-\int_{\Sigma} d \mu \phi^{2}\left\langle W, \mathcal{L}^{\Sigma} W\right\rangle
$$

Then, using (1.8) we have that

$$
0 \leq \int_{\Sigma} d \mu|W|^{2}|\nabla \phi|^{2}+\int_{\Sigma} d \mu \phi^{2} A^{N_{\beta}}(W, i) A^{N_{\beta}}(i, W)-\frac{1}{2} \int_{\Sigma} d \mu \phi^{2}|W|^{2} .
$$

Let $M=\sup _{x \in \Sigma,|v|=1} A^{N_{\beta}}(v, i) A^{N_{\beta}}(i, v)$. Using standard cut-off functions of increasing domain and $\left|\nabla^{\Sigma} \phi\right|^{2} \leq 1$, we get

$$
0 \leq\left(M-\frac{1}{2}\right) \int_{\Sigma} d \mu|W|^{2}
$$

Since $W \not \equiv 0$, we get the theorem.

In our next application, we will need to make use of the inequality

$$
2 A^{N_{\alpha}}\left(W, e_{i}\right) A^{N_{\alpha}}\left(e_{i}, W\right)-|A|^{2}|W|^{2} \leq|W|^{2} \sup _{p \in \Sigma} \inf _{\left\{N_{\alpha}(p)\right\}} \sum_{\alpha}\left|\kappa_{\alpha 1}^{2}-\kappa_{\alpha 2}^{2}\right| .
$$


This can be realized by diagonalizing $A^{N_{\alpha}}$ for each vector in a normal frame $\left\{N_{\alpha}\right\}$. We then get

$$
\begin{aligned}
2 A^{N_{\alpha}}\left(W, e_{i}\right) A^{N_{\alpha}}\left(e_{i}, W\right)-|A|^{2}|W|^{2} & =\sum_{\alpha} 2\left(\sum_{i} \kappa_{\alpha i}^{2} W_{\alpha i}^{2}\right)-\left(\kappa_{\alpha 1}^{2}+\kappa_{\alpha 2}^{2}\right)\left(W_{\alpha 1}^{2}+W_{\alpha 2}^{2}\right) \\
& =\sum_{\alpha} \sum_{i \neq j}\left(\kappa_{\alpha i}^{2}-\kappa_{\alpha j}^{2}\right) W_{\alpha i}^{2} \\
& \leq \sum_{\alpha}\left|\kappa_{\alpha 1}^{2}-\kappa_{\alpha 2}^{2}\right||W|^{2} .
\end{aligned}
$$

Note that we are free to choose $\left\{N_{\alpha}\right\}$ at any point, so therefore we get (2.8).

As in Fischer-Colbrie [4, the index of the operator $L$ acting on scalar functions on $B_{R} \subset \Sigma$ is increasing in $R$ for any exhaustion of $\Sigma$ by $B_{R}$. The index of $L$ on $\Sigma$ is defined to be $\operatorname{Index}_{\Sigma}(L)=\sup _{R}\left(\operatorname{Index}_{B_{R}}(L)\right)$.

Following the work of Ros [6] and Urbano [7] on the Jacobi operator on minimal surfaces, we may give lower bounds for the index of $L$ if we have a condition on the principal curvatures. That is, we have

Theorem 2.2. Let $\Sigma$ be a two-dimensional orientable self-shrinker of polynomial volume growth immersed in $\mathbb{R}^{n}$ with genus $g$ and principal curvatures $\kappa_{\alpha i}$ for a normal frame $\left\{N_{\alpha}\right\}$. If $\sup _{p \in \Sigma} \inf _{\left\{N_{\alpha}(p)\right\}} \sum_{\alpha}\left|\kappa_{\alpha 1}^{2}-\kappa_{\alpha 2}^{2}\right| \leq \delta<1$, then the index of $L$ acting on scalar functions of $\Sigma$ has a lower bound given by

$$
\operatorname{Index}_{\Sigma}(L) \geq \frac{g}{n} .
$$

Proof. We may assume $\operatorname{Index}_{\Sigma}(L)=J<\infty$. As in Fischer-Colbrie [4, there exist $L_{\mu}^{2}(\Sigma)$ functions $\psi_{1}, \ldots, \psi_{J}$ such that if $f \in C_{0}^{\infty}(\Sigma)$ and $\int_{\Sigma} d \mu f \psi_{i}=0$ for all $i$, then $-\int_{\Sigma} d \mu f L f \geq 0$.

Similar to Farkas-Kra [3, p. 42], we have $g$ linearly independent $L_{\mu}^{2}(\Sigma)$ GHF's $\omega_{i}$ with dual vectors $W_{i}$. For now, consider the case that $g<\infty$ and define $V=\operatorname{span}\left\{W_{i}\right\}$ where we are considering $W_{i}$ to be vector fields with values in $\mathbb{R}^{n}$. Consider any $\phi \in C_{0}^{\infty}(\Sigma)$. Similar to the calculation in Theorem 2.1, we have that for any $W \in V$ that

$$
-\int_{\Sigma} d \mu\left\langle\phi W, L^{E}(\phi W)\right\rangle=\int_{\Sigma} d \mu|W|^{2}|\nabla \phi|^{2}-\int_{\Sigma} d \mu \phi^{2}\left\langle W, L^{E} W\right\rangle .
$$

Using (1.9) and (2.8), we get that

$$
\begin{aligned}
\int_{\Sigma} d \mu \phi^{2}\left\langle W, L^{E} W\right\rangle & \geq \int_{\Sigma} d \mu \phi^{2}|W|^{2}\left(1-\sup _{p \in \Sigma} \inf _{\left\{N_{\alpha}(p)\right\}} \sum_{\alpha}\left|\kappa_{\alpha 1}^{2}-\kappa_{\alpha 2}^{2}\right|\right) \\
& \geq(1-\delta) \int_{\Sigma} d \mu \phi^{2}|W|^{2} .
\end{aligned}
$$

Since $\operatorname{dim} V<\infty$, by using a standard cut-off function of large enough domain and $|\nabla \phi|^{2} \leq 1$, we may ensure that $\operatorname{dim} V=\operatorname{dim} \phi V$ and that $-\int_{\Sigma} d \mu\left\langle\phi W, L^{E}(\phi W)\right\rangle$ $<0$ for all $0 \neq \phi W \in \phi V$.

We consider the linear map $F: \phi V \rightarrow \mathbb{R}^{n J}$ given by

$$
F(\phi W)=\left(\int_{\Sigma} d \mu \phi W \psi_{1}, \ldots, \int_{\Sigma} d \mu \phi W \psi_{J}\right) .
$$


For any $\phi W \in \phi V$, if $\phi W \in \operatorname{Ker} F$, then each of its coordinate functions is orthogonal to every $\psi_{i}$. Therefore, $-\int_{\Sigma} d \mu\left\langle\phi W, L^{E} \phi W\right\rangle \geq 0$. Hence, we have that $\phi W \equiv 0$. Therefore, $\operatorname{Ker} F=0$.

So $g=\operatorname{dim} V \leq n J$, and we have the conclusion for the case of $g<\infty$. For the case of $g=\infty$, it is clear that the above argument shows that $3 J \geq m$ for all integers $m$.

\section{Applications to Lowest Eigenvalue $\eta_{0}$}

For any non-compact manifold $\Sigma$ the operator $L$ on scalar functions may not have a nice spectrum, but we may still define the lowest eigenvalue of $L$ by

$$
\eta_{0} \equiv \inf _{\phi \in C_{0}^{\infty}(\Sigma)} \frac{\int_{\Sigma} d \mu\left(|\nabla \phi|^{2}-|A|^{2} \phi^{2}-\frac{1}{2} \phi^{2}\right)}{\int_{\Sigma} d \mu \phi^{2}} .
$$

We get upper bounds for $\eta_{0}$.

Theorem 3.1. Let $\Sigma$ be a two-dimensional orientable self-shrinker of polynomial volume growth immersed in $\mathbb{R}^{n}$ with genus $\geq 1$. The lowest eigenvalue of $L$ acting on scalar functions on $\Sigma$ has two upper bounds given by

$$
\begin{aligned}
& \eta_{0} \leq-1+\sup _{x \in \Sigma,|v|=1} A^{N_{\beta}}(v, i) A^{N_{\beta}}(i, v) \\
& \eta_{0} \leq-1+\sup _{p \in \Sigma} \inf _{\left\{N_{\alpha}(p)\right\}} \sum_{\alpha}\left|\kappa_{\alpha 1}^{2}-\kappa_{\alpha 2}^{2}\right| .
\end{aligned}
$$

Proof. Let $\phi \in C_{0}^{\infty}(\Sigma)$ and $\omega$ be any non-zero GHF on $\Sigma$ with daul vector field $W$. Also, let $M_{p}=\sup _{v \in T_{p} \Sigma,|v|=1} A^{N_{\beta}}(v, i) A^{N_{\beta}}(i, v)$. Note that $M_{p}$ depends on $p \in \Sigma$ and is not the supremum over $\Sigma$. Consider the tangent vector field $\phi W$. Plugging the coordinate functions of $\phi W$ into the definition of $\eta_{0}$ we get

$$
\eta_{0} \int_{\Sigma} d \mu \phi^{2}|W|^{2} \leq \int_{\Sigma} d \mu\left(\left|\nabla^{E}(\phi W)\right|^{2}-|A|^{2} \phi^{2}|W|^{2}-\frac{1}{2} \phi^{2}|W|^{2}\right) .
$$

Note that our expression involves the Euclidean connection $\nabla^{E}$. As in the proof of Theorem 2.1, we have that

$$
\int_{\Sigma} d \mu\left(\left|\nabla^{E}(\phi W)\right|^{2}=\int_{\Sigma} d \mu|\nabla \phi|^{2}|W|^{2}-\int_{\Sigma} d \mu \phi^{2}\left\langle W, \mathcal{L}^{E} W\right\rangle .\right.
$$

Now using (1.9) we have that $-\int_{\Sigma} d \mu \phi^{2}\left\langle W, \mathcal{L}^{E} W\right\rangle \leq \int_{\Sigma} d \mu \phi^{2}|W|^{2}\left(2 M_{p}-1 / 2\right)$. Then, using standard cut-off functions of increasing domain and $\left|\nabla^{\Sigma} \phi\right|^{2} \leq 1$, we get that

$$
\eta_{0} \int_{\Sigma} d \mu|W|^{2} \leq \int_{\Sigma} d \mu|W|^{2}\left(2 M_{p}-|A|^{2}-1\right) .
$$

Using that $M_{p}-|A|^{2}(p) \leq 0$, we get (3.2).

Similarly, if we choose an orthonormal basis $\left\{N_{\alpha}\right\}$ and analyze our terms differently, then from (1.9) we get

$$
\eta_{0} \int_{\Sigma} d \mu \phi^{2}|W|^{2} \leq \int_{\Sigma} d \mu \phi^{2}\left(2 A^{N_{\alpha}}\left(W, e_{i}\right) A^{N_{\alpha}}\left(e_{i}, W\right)-|A|^{2}|W|^{2}-|W|^{2}\right) .
$$

So, using (2.8) we get (3.3). 
For the case of compact $\Sigma$ with genus $\geq 1$ we may give another bound for the lowest eigenvalue of $L$ since it is actually realized by a funtion $u>0$. This allows us to use $\mathcal{L}^{\Sigma}$, instead of $\mathcal{L}^{E}$, to get better bounds.

Theorem 3.2. Let $\Sigma$ be a two-dimensional orientable compact self-shrinker immersed in $\mathbb{R}^{n}$ with genus $g \geq 1$ and principal curvatures $\kappa_{\alpha i}$ in a normal frame $\left\{N_{\alpha}\right\}$. Let $\eta_{0}$ be the lowest eigenvalue of $L$ acting on scalar functions on $\Sigma$. We have that

$$
\eta_{0} \leq-3 / 2+\sup _{p \in \Sigma} \inf _{\left\{N_{\alpha}(p)\right\}} \sum_{\alpha}\left|\kappa_{\alpha 1}^{2}-\kappa_{\alpha 2}^{2}\right| .
$$

Proof. Let $u$ be the eigenfunction for the lowest eigenvalue $\eta_{0}$. Note that by standard theory, $u>0$. Let $\omega$ be a GHF with $W$ its dual vector field. Consider the equation

$$
\int_{\Sigma} d \mu|W|^{2} L u=-\eta_{0} \int_{\Sigma} d \mu|W|^{2} u
$$

We perform integration by parts on the left hand side; use that

$$
L|W|^{2} \geq 2\left\langle W, \mathcal{L}^{\Sigma} W\right\rangle+\left(|A|^{2}+\frac{1}{2}\right)|W|^{2},
$$

(1.8), and (2.8) to get

$$
-\eta_{0} \int_{\Sigma} d \mu|W|^{2} u \geq \int_{\Sigma} d \mu \frac{3}{2}|W|^{2} u-\int_{\Sigma} d \mu|W|^{2} u \sup _{p \in \Sigma} \inf _{\left\{N_{\alpha}(p)\right\}} \sum_{\alpha}\left|\kappa_{\alpha 1}^{2}-\kappa_{\alpha 2}^{2}\right| .
$$

Since $|W|^{2} u \geq 0$ and $|W|^{2} u \not \equiv 0$ we get (3.8).

\section{ACKNOWLEDGEMENTS}

The author would like to thank Professor William Minicozzi and Professor Joel Spruck for their guidance and support.

\section{REFERENCES}

[1] Huai-Dong Cao and Haizhong Li, A gap theorem for self-shrinkers of the mean curvature flow in arbitrary codimension, Calc. Var. Partial Differential Equations 46 (2013), no. 3-4, 879-889, DOI 10.1007/s00526-012-0508-1. MR3018176

[2] Tobias H. Colding and William P. Minicozzi II, Generic mean curvature flow I: generic singularities, Ann. of Math. (2) 175 (2012), no. 2, 755-833, DOI 10.4007/annals.2012.175.2.7. MR.2993752

[3] H. M. Farkas and I. Kra, Riemann surfaces, 2nd ed., Graduate Texts in Mathematics, vol. 71, Springer-Verlag, New York, 1992. MR1139765 (93a:30047)

[4] D. Fischer-Colbrie, On complete minimal surfaces with finite Morse index in three-manifolds, Invent. Math. 82 (1985), no. 1, 121-132, DOI 10.1007/BF01394782. MR808112 (87b:53090)

[5] Jürgen Jost, Riemannian geometry and geometric analysis, 6th ed., Universitext, Springer, Heidelberg, 2011. MR2829653

[6] Antonio Ros, One-sided complete stable minimal surfaces, J. Differential Geom. 74 (2006), no. 1, 69-92. MR2260928 (2007g:53008)

[7] Francisco Urbano, Second variation of one-sided complete minimal surfaces, Rev. Mat. Iberoam. 29 (2013), no. 2, 479-494, DOI 10.4171/RMI/727. MR3047425

Department of Mathematics, Johns Hopkins University, 3400 North Charles Street, Baltimore, Maryland 21218-2686

Current address: Department of Mathematics, University of Washington, Box 354350, Seattle, Washington 9815-4350

E-mail address: mmcgona1@math.washington.edu 